\title{
Long-Term Impact of Diabetes Mellitus on Initially Conservatively Managed Patients With Severe Aortic Stenosis
}

\author{
Xiaoyang Song, MD; Naritatsu Saito, MD; Takeshi Morimoto, MD; Tomohiko Taniguchi, MD; \\ Hiroki Shiomi, MD; Kenji Ando, MD; Kazuya Nagao, MD; Norio Kanamori, MD; \\ Koichiro Murata, MD; Takeshi Kitai, MD; Yuichi Kawase, MD; Chisato Izumi, MD; \\ Makoto Miyake, MD; Hirokazu Mitsuoka, MD; Masashi Kato, MD; Yutaka Hirano, MD; \\ Shintaro Matsuda, MD; Tsukasa Inada, MD; Tomoyuki Murakami, MD; Yasuyo Takeuchi, MD; \\ Keiichiro Yamane, MD; Mamoru Toyofuku, MD; Mitsuru Ishii, MD; Eri Minamino-Muta, MD; \\ Takao Kato, MD; Yusuke Yoshikawa, MD; Moriaki Inoko, MD; Tomoyuki Ikeda, MD; \\ Katsuhisa Ishii, MD; Kozo Hotta, MD; Nobuya Higashitani, MD; Yoshihiro Kato, MD; \\ Yasutaka Inuzuka, MD; Chiyo Maeda, MD; Toshikazu Jinnai, MD; Yuko Morikami, MD; \\ Kenji Minatoya, MD; Takeshi Kimura, MD on behalf of the CURRENT AS Registry Investigators
}

\begin{abstract}
Background: Although diabetes mellitus (DM) is a common comorbidity of aortic stenosis (AS), clinical evidence about the long-term effect of DM on patients with AS is insufficient.

Methods and Results: Data were acquired from CURRENT AS, a large Japanese multicenter registry that enrolled 3,815 patients with severe AS. Patients without initial valve replacement were defined as the conservative group; among them, 621 (23.4\%) had DM, whereas 1997 did not. The DM group was further divided into 2 groups according to insulin treatment (insulin-treated DM, $\mathrm{n}=130$; non-insulin treated DM, $n=491$ ). The primary outcome was a composite of aortic valve (AV)-related death and heart failure (HF) hospitalization. Secondary outcomes were AV-related death, HF hospitalization, all-cause death, cardiovascular death, sudden death, and surgical or transcatheter AV replacement during follow up. As a result, DM was associated with higher risk for the primary outcome (52.8\% vs. $42.9 \%, P<0.001$ ), with a statistically significant adjusted hazard ratio (HR 1.33, 95\% confidence interval: $1.14-1.56$, $\mathrm{P}<0.001)$. All secondary outcomes were not significantly different between DM and non-DM patients after adjusting for confounding factors, except for HF hospitalization. Insulin use was not associated with higher incidence of primary or secondary outcome.
\end{abstract}

Conclusions: In initially conservatively managed patients with AS, DM was independently associated with higher risk for a composite of AV-related death or HF hospitalization; however, insulin use was not associated with poor outcomes.

Key Words: Aortic stenosis; Diabetes mellitus; Survival analysis

A ortic stenosis (AS) and diabetes mellitus (DM) are both comorbidities frequently observed in aging populations. In a nation-wide study in Spain, 78,223 patients underwent surgical aortic valve replacement (SAVR); $23.5 \%$ of them had Type 2 DM. ${ }^{1}$ In the Placement of Aortic Transcatheter Valves (PARTNER) 3 trial, ${ }^{2}$ $30.8 \%$ of patients had DM at baseline. Also, Nishimura et $\mathrm{al}^{3}$ analyzed 140 asymptomatic patients with severe AS;
$21 \%$ were diabetic. Cumulative evidence suggested that DM caused damage to the aortic valve in a similar mechanism to atherosclerosis. ${ }^{4}$ As previous studies reported, hyperglycemia, oxidative stress, insulin resistance and elevated inflammation, induced alternations not only in endothelial cells but also in the whole valve.,56 Therefore, DM may play an important role in the development and progression of AS. In addition, clinical data from observational studies

Received June 25, 2020; revised manuscript received September 2, 2020; accepted September 7, 2020; J-STAGE Advance Publication released online November 18, 2020 Time for primary review: 20 days

Cardiovascular Medicine, Kyoto University Graduate School of Medicine, Kyoto (X.S., N.S., H.S., S.M., E.M.-M., T. Kato, Y.Y., T. Kimura); Department of Clinical Epidemiology, Hyogo College of Medicine, Nishinomiya (T. Morimoto); Department of Cardiology, Kokura Memorial Hospital, Kitakyusyu (T.T., K.A.); Division of Cardiology, Cardiovascular Center, Osaka Red Cross Hospital, Osaka (K.N., T. Inada); Shimada Municipal Hospital, Shimada (N.K.); Department of Cardiology, Shizuoka City Shizuoka Hospital, Shizuoka (K. Murata); Department of Cardiovascular Medicine, Kobe City Medical Center General Hospital, Kobe (T. Kitai); Department of Cardiovascular Medicine, Kurashiki Central Hospital, Kurashiki (Y. Kawase); Department of Cardiology, Tenri Hospital, Tenri (C.I., M.M.); Division of Cardiology, Nara Hospital, Kindai University Faculty of Medicine, Ikoma (H.M.); Department of Cardiology, Mitsubishi Kyoto Hospital, Kyoto (M.K.); Department of Cardiology, Kindai University Hospital, Osakasayama (Y.H.); Department of Cardiology, Koto Memorial Hospital, Higashiomi (T. Murakami); Department of Cardiology, Shizuoka General Hospital, Shizuoka (Y.T.); Department of Cardiology, Nishikobe Medical Center, Kobe (K.Y.); (Footnote continued the next page.) 


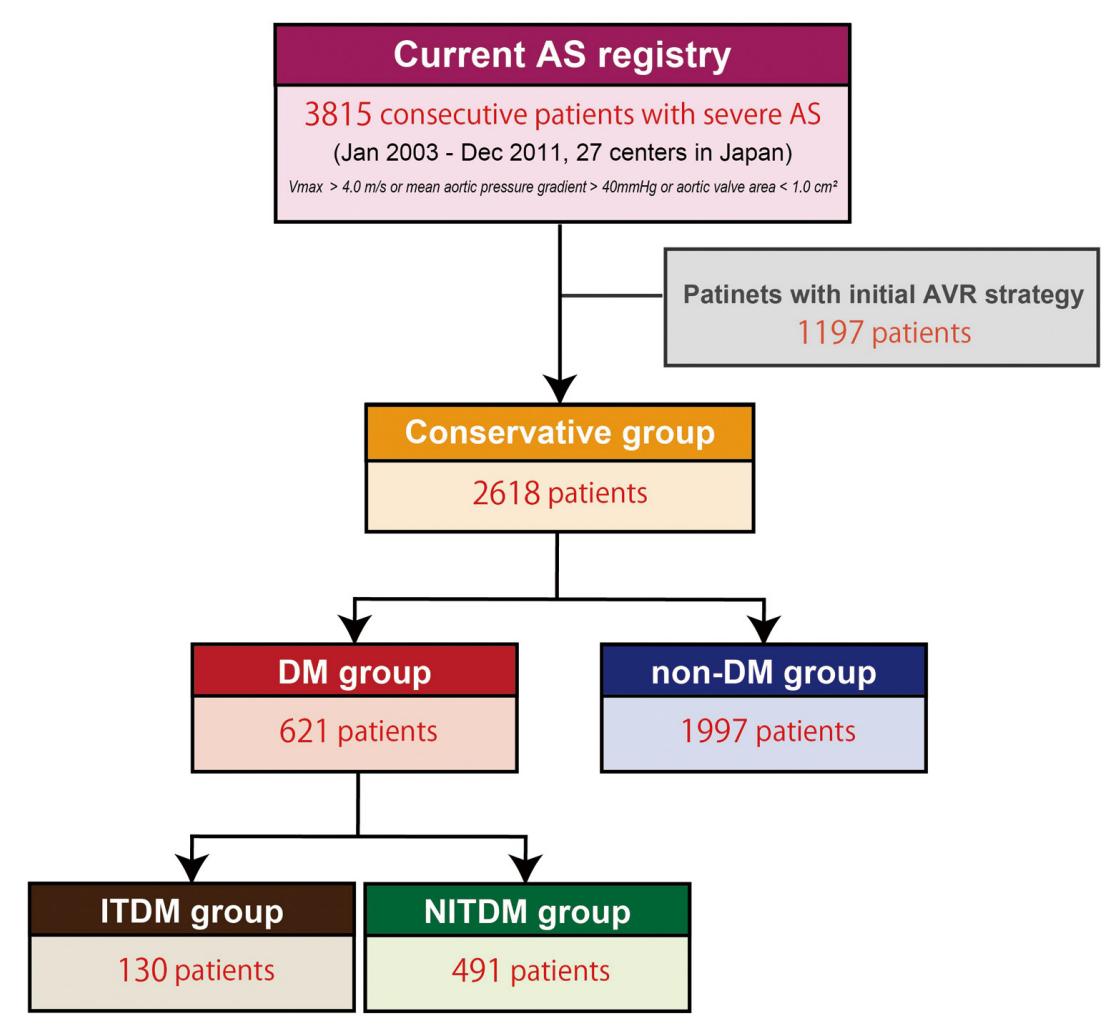

Figure 1. Study flow chart. AVR, aortic valve replacement; DM, diabetes mellitus; ITDM, insulin-treated DM.

revealed that DM was associated with increased risk of aortic valve calcification. , $^{\mathbf{8}}$ However, opinions are divided over whether DM accelerates the progression of $\mathrm{AS}^{\mathbf{6}, 9}$ or not. ${ }^{10}$

Although surgical or interventional aortic valve replacement (SAVR/TAVR) was recommended for patients with severe AS, ${ }^{11}$ there are still patients who do not undergo a replacement due to advanced age, comorbidities or patient's values. ${ }^{12}$ Several studies have investigated the prognosis these patients. ${ }^{13,14}$ However, the effect of DM still remains unclear. Therefore, we sought to clarify the effect of DM on the clinical outcomes of initially conservatively managed patients with severe AS by comparing those with or without $\mathrm{DM}$ in a large Japanese multicenter registry.

\section{Methods}

\section{Study Population}

A retrospective study was performed using data obtained from a multicenter Contemporary Outcomes After Surgery and Medical Treatment in Patients with Severe Aortic Stenosis (CURRENT AS) registry, which enrolled 3,815 consecutive patients with severe AS. Characteristics of the CURRENT AS registry have been described previously. ${ }^{15}$ Briefly, data of patients with severe AS from 27 centers in Japan (Supplementary File) were collected from January 2003 to December 2011.

Patients who met the definition of severe AS (peak aortic jet velocity $>4.0 \mathrm{~m} / \mathrm{s}$; mean aortic pressure gradient $>40 \mathrm{mmHg}$; aortic valve area $<1.0 \mathrm{~cm}^{2}$ ) were enrolled in the cohort. In this study, the initial conservative group was defined as patients who did not receive aortic valve replacement initially. As a result, 3,815 patients were enrolled, and data were extracted from 2,618 patients in the initial conservative group. Among them, 621 had DM (DM group), whereas 1,997 patients had no DM (non-DM group) (Figure 1). The DM group was further divided into 2 groups according to insulin treatment; insulin-treated DM (ITDM) was defined as DM treated with insulin therapy $(n=130)$, and

Department of Cardiology, Japanese Red Cross Wakayama Medical Center, Wakayama (M.T.); Department of Cardiology, National Hospital Organization Kyoto Medical Center, Kyoto (M. Ishii); Cardiovascular Center, The Tazuke Kofukai Medical Research Institute, Kitano Hospital, Osaka (M. Inoko); Department of Cardiology, Hikone Municipal Hospital, Hikone (T. Ikeda); Department of Cardiology, Kansai Electric Power Hospital, Osaka (K.I.); Department of Cardiology, Hyogo Prefectural Amagasaki General Medical Center, Amagasaki (K.H.); Department of Cardiology, Japanese Red Cross Otsu Hospital, Otsu (N.H., T.J.); Department of Cardiology, Saiseikai Noe Hospital, Osaka (Y. Kato); Department of Cardiology, Shiga Medical Center for Adults, Moriyama (Y.I.); Department of Cardiology, Hamamatsu Rosai Hospital, Hamamatsu (C.M.); Department of Cardiology, Hirakata Kohsai Hospital, Hirakata (Y.M.); and the Department of Cardiovascular Surgery, Kyoto University Graduate School of Medicine, Kyoto (K. Minatoya), Japan

Mailing address: Naritatsu Saito, MD, Department of Cardiovascular Medicine, Kyoto University Graduate School of Medicine, 54 Shogoin Kawahara-cho, Sakyo-ku, Kyoto 606-8507, Japan. E-mail: naritatu@kuhp.kyoto-u.ac.jp

All rights are reserved to the Japanese Circulation Society. For permissions, please e-mail: cj@j-circ.or.jp ISSN-1346-9843 
Table 1. Baseline Characteristics of the DM and Non-DM Group

\begin{tabular}{|c|c|c|c|}
\hline Clinical characteristics & $\begin{array}{l}\text { DM group } \\
(n=621)\end{array}$ & $\begin{array}{c}\text { Non-DM group } \\
(n=1,997)\end{array}$ & $P$ value \\
\hline Age, years & $78.1 \pm 8.1$ & $80.3 \pm 9.8$ & $<0.001$ \\
\hline Age $\geq 80$ years $^{\dagger}$ & $274(44.1)$ & $1,156(57.9)$ & $<0.001$ \\
\hline Men $^{\dagger}$ & $270(43.5)$ & $666(33.4)$ & $<0.001$ \\
\hline $\mathrm{BMI}<22^{\dagger}$ & $345(55.6)$ & $1,359(68.1)$ & $<0.001$ \\
\hline $\mathrm{BSA}, \mathrm{m}^{2}$ & $1.49 \pm 0.18$ & $1.42 \pm 0.18$ & $<0.001$ \\
\hline STS score (PROM), \% & $5.1(3.3-9.5)$ & $4.0(2.4-6.9)$ & $<0.001$ \\
\hline Insulin use & $130(20.9)$ & $0(0.0)$ & \\
\hline Hypertension ${ }^{\dagger}$ & $511(82.3)$ & $1,349(67.6)$ & $<0.001$ \\
\hline Dyslipidemia & $299(48.2)$ & $552(27.6)$ & $<0.001$ \\
\hline With statin therapy & $230(37.0)$ & $401(20.1)$ & $<0.001$ \\
\hline Smoking & $157(25.3)$ & $359(18.0)$ & $<0.001$ \\
\hline Current smoking ${ }^{\dagger}$ & $26(4.2)$ & $87(4.4)$ & 0.86 \\
\hline With symptom & $246(39.6)$ & $854(42.8)$ & 0.16 \\
\hline Chest pain & 56 & 151 & \\
\hline Syncope & 16 & 72 & \\
\hline $\mathrm{HF}$ & 207 & 737 & \\
\hline Serum creatinine level $>0.83 \mathrm{mg} / \mathrm{dL}^{\dagger}$ & $382(61.5)$ & $949(47.5)$ & $<0.001$ \\
\hline Hemodialysis $^{\dagger}$ & $95(15.3)$ & $175(8.8)$ & $<0.001$ \\
\hline Anemia $^{\dagger}$ & $382(61.5)$ & $1,106(55.4)$ & 0.007 \\
\hline Acute HF requiring hospitalization ${ }^{\dagger}$ & $118(19.0)$ & $402(20.1)$ & 0.54 \\
\hline Prior myocardial infarction ${ }^{\dagger}$ & $121(19.5)$ & $151(7.6)$ & $<0.001$ \\
\hline Prior symptomatic stroke ${ }^{\dagger}$ & $115(18.5)$ & $281(14.1)$ & 0.0069 \\
\hline Atrial fibrillation ${ }^{\dagger}$ & $134(21.6)$ & $487(24.4)$ & 0.15 \\
\hline Any combined valvular disease (moderate or severe) ${ }^{\dagger}$ & $197(31.7)$ & $882(44.2)$ & $<0.001$ \\
\hline Aortic/peripheral vascular disease requiring treatment ${ }^{\dagger}$ & $76(12.2)$ & $136(6.8)$ & $<0.001$ \\
\hline Chronic lung disease & $58(9.3)$ & $201(10.1)$ & 0.60 \\
\hline Chronic lung disease (moderate or severe) ${ }^{\dagger}$ & $20(3.2)$ & $73(3.7)$ & 0.61 \\
\hline Malignancy & $95(15.3)$ & $291(14.6)$ & 0.66 \\
\hline Malignancy currently under treatment ${ }^{\dagger}$ & $33(5.3)$ & $92(4.6)$ & 0.47 \\
\hline Liver cirrhosis $^{\dagger}$ & $9(1.5)$ & $23(1.2)$ & 0.56 \\
\hline Prior $\mathrm{PCl}^{\dagger}$ & $169(27.2)$ & $230(11.5)$ & $<0.001$ \\
\hline Prior CABG & $79(12.7)$ & $88(4.4)$ & $<0.001$ \\
\hline \multicolumn{4}{|l|}{ Echocardiographic variables } \\
\hline $\mathrm{V}_{\max }, \mathrm{m} / \mathrm{s}$ & $3.7 \pm 0.7$ & $3.9 \pm 0.9$ & $<0.001$ \\
\hline $\mathrm{V}_{\max } \geq 4 \mathrm{~m} / \mathrm{s}^{\dagger}$ & $217(34.9)$ & $974(48.8)$ & $<0.001$ \\
\hline Peak aortic PG, mmHg & $55.6 \pm 22.4$ & $65.1 \pm 28.3$ & $<0.001$ \\
\hline Mean aortic $\mathrm{PG}, \mathrm{mmHg}$ & $30.8 \pm 13.0$ & $36.7 \pm 17.9$ & $<0.001$ \\
\hline AVA (equation of continuity), $\mathrm{cm}^{2}$ & $0.77 \pm 0.17$ & $0.74 \pm 0.18$ & $<0.001$ \\
\hline AVA index, $\mathrm{cm}^{2} / \mathrm{m}^{2}$ & $0.53 \pm 0.11$ & $0.53 \pm 0.13$ & 0.82 \\
\hline LVEF, \% & $60.3 \pm 14.0$ & $63.6 \pm 12.9$ & $<0.001$ \\
\hline$<40$ & $63(10.1)$ & $127(6.4)$ & 0.002 \\
\hline$<50$ & $127(20.5)$ & $261(13.1)$ & $<0.001$ \\
\hline$<68^{\dagger}$ & $413(66.5)$ & $1,161(58.1)$ & $<0.001$ \\
\hline LVSD, mm & $31.4 \pm 8.1$ & $29.4 \pm 7.3$ & $<0.001$ \\
\hline LVDD, mm & $46.6 \pm 6.9$ & $45.0 \pm 6.8$ & $<0.001$ \\
\hline IVST in diastole, mm & $10.9 \pm 2.0$ & $11.1 \pm 2.3$ & 0.009 \\
\hline PWT in diastole, $\mathrm{mm}$ & $10.5 \pm 1.9$ & $10.7 \pm 1.9$ & 0.056 \\
\hline Moderate or severe AR & $74(11.9)$ & $423(21.2)$ & $<0.001$ \\
\hline Moderate or severe MR & $112(18.0)$ & $424(21.2)$ & 0.08 \\
\hline Moderate or severe MS & $15(2.4)$ & $67(3.4)$ & 0.24 \\
\hline Moderate or severe TR & $83(13.4)$ & 398 (19.9) & $<0.001$ \\
\hline TR pressure $\geq 40 \mathrm{mmHg}^{\dagger}$ & $81(13.0)$ & $345(17.3)$ & 0.013 \\
\hline
\end{tabular}

Values are presented as number (\%), mean $\pm \mathrm{SD}$, or median (interquartile range). AR, aortic regurgitation; $\mathrm{AVA}$, aortic valve area; $\mathrm{BMI}$, body mass index; BSA, body surface area; CABG, coronary artery bypass grafting; DM, diabetes mellitus; HF, heart failure; IVST, interventricular septum thickness; LV, left ventricular; LVDD, left ventricular end-diastolic dimension; LVEF, left ventricular ejection fraction; LVSD, left ventricular end-systolic dimension; MR, mitral regurgitation; MS, mitral stenosis; PCl, percutaneous coronary intervention; PG, pressure gradient; PROM, predicted risk of mortality; PWT, posterior wall thickness; STS, Society of Thoracic Surgeons; TR, tricuspid regurgitation; Vmax, peak aortic jet velocity. Variables selected for Cox proportional hazard model. 
Table 2. Baseline Characteristics of the ITDM and Non-ITDM Group

\begin{tabular}{|c|c|c|c|}
\hline Clinical characteristics & $\begin{array}{l}\text { ITDM group } \\
(n=130)\end{array}$ & $\begin{array}{c}\text { Non-ITDM group } \\
(n=491)\end{array}$ & $P$ value \\
\hline Age, years & $77.5 \pm 8.6$ & $78.2 \pm 8.0$ & 0.36 \\
\hline Age $\geq 80$ years ${ }^{\dagger, \ddagger}$ & $55(42.3)$ & $219(44.6)$ & 0.64 \\
\hline Ment, & $58(44.6)$ & $212(43.2)$ & 0.77 \\
\hline $\mathrm{BMI}<22^{\dagger, \ddagger}$ & $70(53.9)$ & $275(56.0)$ & 0.66 \\
\hline $\mathrm{BSA}, \mathrm{m}^{2}$ & $1.48 \pm 0.19$ & $1.49 \pm 0.18$ & 0.50 \\
\hline STS score (PROM), \% & $4.8(3.0-8.8)$ & $7.1(4.0-11.7)$ & $<0.001$ \\
\hline Hypertension $^{\dagger}$ & $106(81.5)$ & $405(82.5)$ & 0.80 \\
\hline Dyslipidemia & $60(46.2)$ & $239(48.7)$ & 0.61 \\
\hline With statin therapy & $43(33.1)$ & $187(38.1)$ & 0.29 \\
\hline Smoking & $39(30.0)$ & $118(24.0)$ & 0.16 \\
\hline Current smoking ${ }^{\dagger}$ & $7(5.4)$ & $19(3.9)$ & 0.44 \\
\hline With symptom & $50(38.5)$ & $196(39.9)$ & 0.76 \\
\hline Chest pain & 11 & 45 & \\
\hline Syncope & 7 & 9 & \\
\hline $\mathrm{HF}$ & 40 & 167 & \\
\hline Serum creatinine level $>0.83 \mathrm{mg} / \mathrm{dL}^{\dagger}$ & $86(66.2)$ & $296(60.3)$ & 0.22 \\
\hline Hemodialysis $^{\dagger}$ & $25(19.2)$ & $70(14.3)$ & 0.16 \\
\hline Anemia $^{\dagger}$ & $98(75.4)$ & $284(57.8)$ & $<0.001$ \\
\hline Acute $\mathrm{HF}$ requiring hospitalization ${ }^{\dagger}$ & $21(16.2)$ & $97(19.8)$ & 0.35 \\
\hline Prior myocardial infarction ${ }^{\dagger, \ddagger}$ & $31(23.9)$ & $90(18.3)$ & 0.16 \\
\hline Prior symptomatic stroke ${ }^{\dagger}$ & $24(18.5)$ & $91(18.5)$ & 0.99 \\
\hline Atrial fibrillation ${ }^{\dagger, \ddagger}$ & $27(20.8)$ & $107(21.8)$ & 0.80 \\
\hline Any combined valvular disease (moderate or severe) ${ }^{\dagger}$ & $29(22.3)$ & $168(34.2)$ & 0.010 \\
\hline Aortic/peripheral vascular disease requiring treatment $t^{t, \neq}$ & $24(18.5)$ & $52(10.6)$ & 0.015 \\
\hline Chronic lung disease & $12(9.2)$ & $46(9.4)$ & 0.96 \\
\hline Chronic lung disease (moderate or severe) ${ }^{\dagger}$ & $4(3.1)$ & $16(3.3)$ & 0.92 \\
\hline Malignancy & $23(17.7)$ & $72(14.7)$ & 0.39 \\
\hline Malignancy currently under treatment ${ }^{\dagger, \neq}$ & $9(6.9)$ & $24(4.9)$ & 0.36 \\
\hline Liver cirrhosis $^{\dagger}$ & $4(3.1)$ & $5(1.0)$ & 0.080 \\
\hline Prior $\mathrm{PCl}^{\dagger}$ & $39(30.0)$ & $130(26.5)$ & 0.42 \\
\hline Prior CABG & $24(18.5)$ & $55(11.2)$ & 0.027 \\
\hline \multicolumn{4}{|l|}{ Echocardiographic variables } \\
\hline $\mathrm{V}_{\max }, \mathrm{m} / \mathrm{s}$ & $3.6 \pm 0.7$ & $3.7 \pm 0.7$ & 0.26 \\
\hline$V_{\max } \geq 4 \mathrm{~m} / \mathrm{s}^{\dagger, \neq}$ & $38(29.2)$ & $179(36.5)$ & 0.12 \\
\hline Peak aortic $\mathrm{PG}, \mathrm{mmHg}$ & $53.7 \pm 21.3$ & $56.2 \pm 22.6$ & 0.26 \\
\hline Mean aortic $\mathrm{PG}, \mathrm{mmHg}$ & $29.4 \pm 12.3$ & $31.1 \pm 13.2$ & 0.21 \\
\hline AVA (equation of continuity), $\mathrm{cm}^{2}$ & $0.78 \pm 0.17$ & $0.77 \pm 0.17$ & 0.52 \\
\hline AVA index, $\mathrm{cm}^{2} / \mathrm{m}^{2}$ & $0.54 \pm 0.11$ & $0.53 \pm 0.11$ & 0.66 \\
\hline LVEF, \% & $59.2 \pm 14.5$ & $60.6 \pm 13.8$ & 0.34 \\
\hline$<40 \%$ & $17(13.1)$ & $46(9.4)$ & 0.21 \\
\hline$<50 \%$ & $28(21.5)$ & $99(20.2)$ & 0.73 \\
\hline$<68 \% \%^{\dagger, \ddagger}$ & $86(66.2)$ & $327(66.6)$ & 0.92 \\
\hline LVSD, mm & $31.4 \pm 8.0$ & $31.5 \pm 8.1$ & 0.95 \\
\hline LVDD, mm & $46.1 \pm 6.9$ & $46.8 \pm 6.9$ & 0.33 \\
\hline IVST in diastole, mm & $10.8 \pm 2.0$ & $10.9 \pm 2.0$ & 0.46 \\
\hline PWT in diastole, $\mathrm{mm}$ & $10.7 \pm 2.0$ & $10.5 \pm 1.9$ & 0.34 \\
\hline Moderate or severe AR & $9(6.9)$ & $65(13.2)$ & 0.048 \\
\hline Moderate or severe MR & $19(14.6)$ & $93(18.9)$ & 0.25 \\
\hline Moderate or severe MS & $1(0.8)$ & $14(2.9)$ & 0.17 \\
\hline Moderate or severe TR & $14(10.8)$ & $69(14.1)$ & 0.33 \\
\hline TR pressure $\geq 40 \mathrm{mmHg}^{\dagger}$ & $19(14.6)$ & $62(12.6)$ & 0.55 \\
\hline
\end{tabular}

Values are presented as number (\%), mean \pm SD, or median (interquartile range). ITDM, insulin-treated DM. Other abbreviations as in Table 1. †Variables selected for Cox proportional hazard model. $\neq$ Variables selected for parsimonious model. 
non-insulin treated DM (NITDM) was defined as DM treated without insulin therapy at baseline $(n=491)$.

The institutional review boards in all 27 participating centers approved the protocol. This study was supervised by the Ethics Committee at Kyoto University, and followed the Declaration of Helsinki. Written informed consent from each patient was waived in this retrospective study because clinical information obtained in routine clinical practice was used, and no patients refused to participate in the study when contacted for follow up.

\section{Data Collection and Definitions}

Baseline clinical data were collected using hospital charts or database review. Angina, syncope, or heart failure (HF) including dyspnea were regarded as AS-related symptoms. Follow-up data were mainly collected by hospital chart review or by contacting patients, relatives, and/or referring physicians via mail with questions regarding survival, symptoms, and subsequent hospitalizations. Patients were considered to have DM if they fulfilled any of the following criteria: (1) fasting glucose level $\geq 126 \mathrm{mg} / \mathrm{dL}$; (2) a random glucose level $\geq 200 \mathrm{mg} / \mathrm{dL}$; (3) $\mathrm{HbAlc} \geq 6.5 \%$; (4) use of glucose-lowering medications; and (5) previous diagnosis of DM.

The primary outcome measure of this study was a composite of the aortic valve (AV)-related death and HF hospitalization. The cause of death was classified according to the Valve Academic Research Consortium (VARC) definitions and was adjudicated by a clinical event committee. ${ }^{16}$ Sudden death was defined as unexplained death in previously stable patients. Aortic valve-related death included aortic procedure-related death, sudden death, and death attributed to HF that was possibly related to the aortic valve. HF hospitalization was defined as hospitalization attributed to worsening HF that required intravenous drug therapy. Secondary outcomes were AV-related death, HF hospitalization, all-cause death, cardiovascular death, sudden death, and surgical or transcatheter aortic valve implantation (SAVR/TAVI) during follow up. Definitions of clinical events have been described in more detail in a previous study. ${ }^{15}$

\section{Statistical Analysis}

Categorical variables were presented as numbers and percentages, and between-group differences were compared using Pearson's chi-squared test or Fisher's exact test. Continuous variables were presented as mean \pm standard deviation or median (interquartile range, IQR) and were compared using the Student's-t test or Wilcoxon rank-sum test according to their distributions. The cumulative incidence of primary and secondary outcomes was investigated using the Kaplan-Meier method, and the difference between DM and non-DM groups was compared using the log-rank test. A Cox proportional hazard model was used to adjust the confounding effects of the differences in baseline characteristics besides DM. Candidate covariates in the Cox proportional hazard models were based on our previous study, and variables included were as follows: DM, insulin use (ITDM), age $\geq 80$ years, male sex, body mass index (BMI) of $<22$, hypertension, acute HF requiring hospitalization, current smoking, hemodialysis, anemia, any combined valvular disease (moderate or severe), previous myocardial infarction (MI), previous symptomatic stroke, previous atrial fibrillation or flutter, aortic/peripheral vascular disease requiring treatment, chronic lung disease

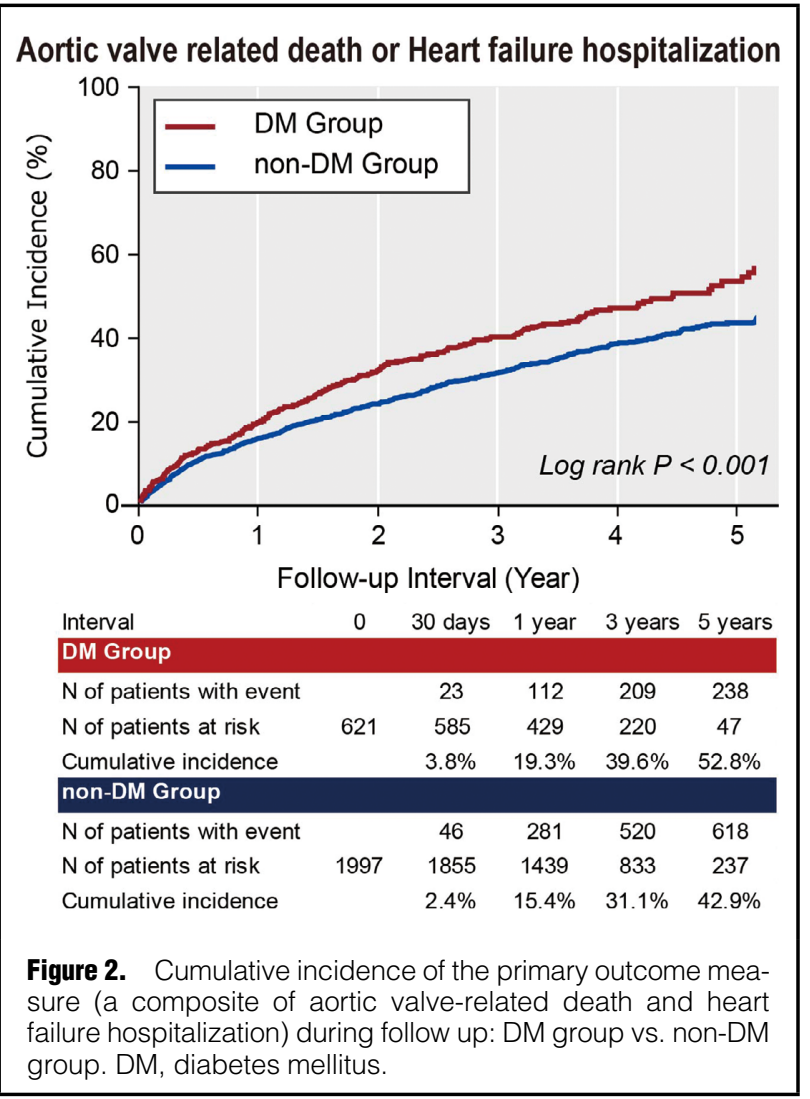

(moderate or severe), malignancy currently under treatment, liver cirrhosis (Child-Pugh B or C), previous percutaneous coronary intervention (PCI) history, ejection fraction $(\mathrm{EF}) \leq 68 \%$, peak aortic jet velocity $\left(\mathrm{V}_{\max }\right)$ of $\geq 4 \mathrm{~m} / \mathrm{s}$, tricuspic regurgitation pressure of $\geq 40 \mathrm{mmHg}$, and serum creatinine $>0.83 \mathrm{mg} / \mathrm{dL}$ (Table 1 and Table 2). For outcomes in which the number of events was too low, we constructed parsimonious model with fewer variables; 10 clinically relevant variables were selected (Table 2). The statistical analysis was conducted by a statistician and a clinician independently. JMP pro 15.1 (SAS Institute Inc., NC, USA) was used to analyze and compare the acquired data. $\mathrm{P}$ values in this study were all 2 -tailed, and a $\mathrm{P}$ value of $<0.05$ was considered statistically significant.

\section{Results}

\section{Baseline Characteristics}

Among the 2,618 patients who were managed with an initial conservative strategy, 621 suffered from DM $(23.7 \%$, DM group), whereas 1,997 had no DM (76.3\%, non-DM group). Significant differences were observed in many aspects between the DM and non-DM groups (Table 1). In brief, patients in the DM group were younger and had a higher BMI and body surface area (BSA). There was no difference in the prevalence of AS-related symptoms (chest pain, syncope, or HF) between the 2 groups. Comorbidities such as hypertension, dyslipidemia, smoking, hemodialysis, anemia and prior MI were more prevalent in the DM group than in the non-DM group. In addition, history of PCI and coronary artery bypass grafting (CABG) was also more common in the DM group than in the non-DM 

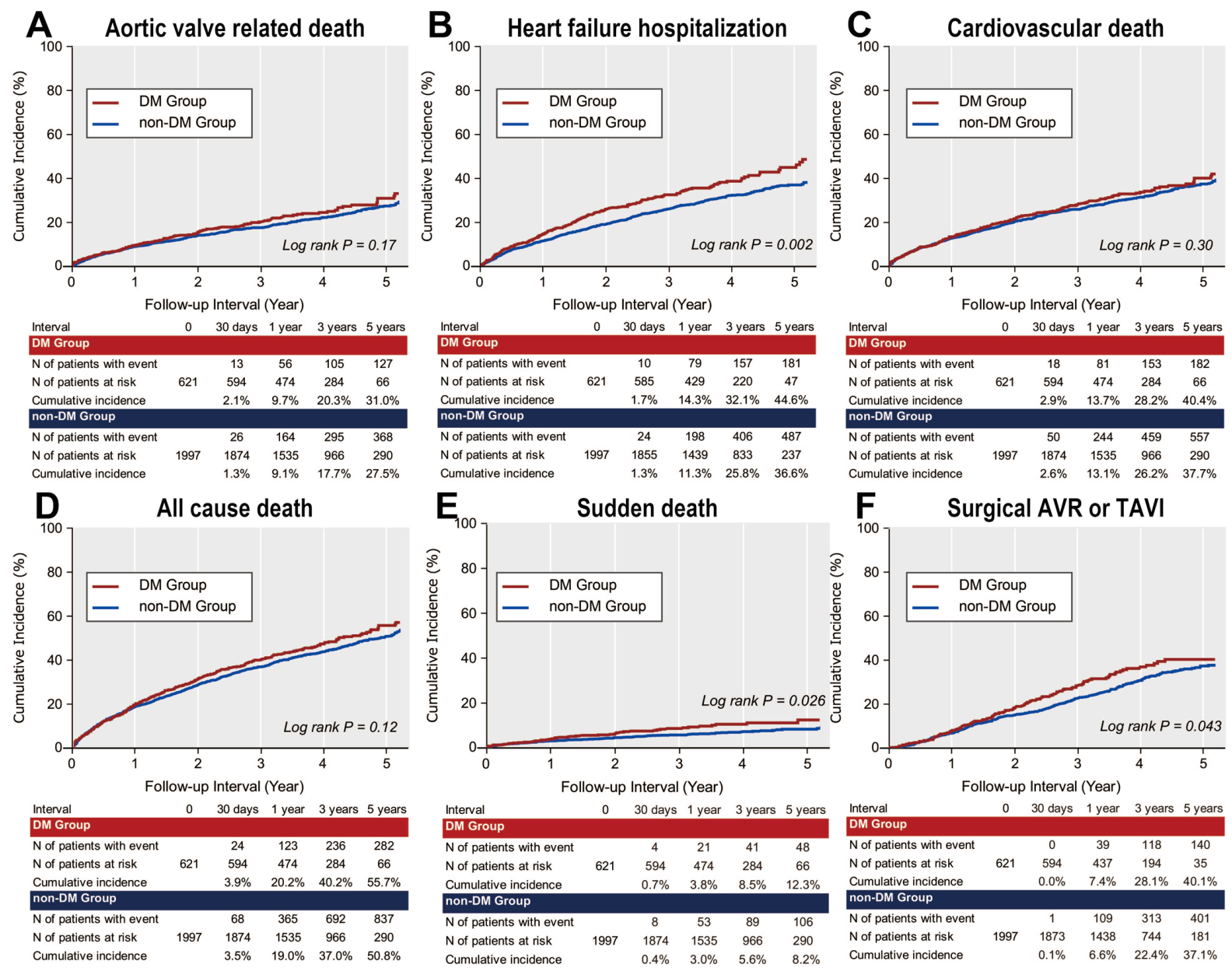

Figure 3. Cumulative incidence of the secondary outcome measures including (A) aortic valve-related death; (B) heart failure hospitalization; (C) cardiovascular death; (D) all-cause death; (E) sudden death; and (F) surgical AVR or TAVI during follow up. DM group vs. non-DM group. DM, diabetes mellitus; AVR, aortic valve replacement; TAVI, transcatheter aortic valve implantation.

group. The difference in some echocardiographic variables was also significant; $V_{\max }$, the mean pressure gradient (mPG), and EF were lower in the DM group. When comparing ITDM and non-ITDM groups, most baseline characteristics were similar; however, the STS (Society of Thoracic Surgeons) score was significantly higher in the ITDM group (Table 2).

\section{Clinical Outcomes}

The median follow-up duration of the surviving patients was 1,062 (IQR, 427-2,070) days. The cumulative 5-year incidence of the primary outcome measure (a composite of AV-related death and HF hospitalization) was significantly higher in the DM group than in the non-DM group $(52.8 \%$ vs. $42.9 \%, \mathrm{P}<0.001$ ) (Figure 2). For the secondary outcomes, the cumulative 5-year incidence of HF hospitalization and sudden death was also significantly higher in the DM group than in the non-DM group $(44.6 \%$ vs. $36.6 \%$, $\mathrm{P}=0.002$; and $12.3 \%$ vs. $8.2 \%, \mathrm{P}=0.03)$. There were no significant differences in the cumulative 5-year incidences of AV-related death, all-cause death and cardiovascular death between the DM and non-DM groups (Figure 3).
The cumulative 5-year incidence of SAVR or TAVI was significantly higher in the DM group than in the non-DM group (40.1\% vs. 37.1\% $\mathrm{P}=0.043$ ) (Figure 3).

The hazard ratio (HR) for primary outcome measure was 1.33 (95\% confidence interval [CI]: $1.13-1.56$; $\mathrm{P}<0.001)$ in the multivariable Cox model, indicating that DM was independently associated with higher risk for AV-related death and HF hospitalization. As for secondary outcomes, after adjusting for confounders, DM was found to be independently associated with higher risk for HF hospitalization (HR 1.36, 95\% CI: 1.14-1.63, P<0.001). However, $\mathrm{DM}$ as compared with non-DM was not associated with higher risk for AV-related death, all-cause death, cardiovascular death and sudden death (Table 3).

When comparing ITDM and non-ITDM groups, the cumulative 5-year incidence of the primary outcome measure was not significantly different between the 2 groups (Figure 4). In the multivariate cox regression analysis, insulin use as compared with no insulin use was not associated with higher risk for AV-related death or HF hospitalization (HR 0.92, 95\% CI: 0.66-1.29, $\mathrm{P}=0.64$ ). For secondary outcomes, no difference was observed in the cumulative 
Table 3. Crude and Adjusted Effects of DM and Insulin Use on Long-Term Clinical Outcomes

\begin{tabular}{|c|c|c|c|c|}
\hline & $\begin{array}{c}\text { Unadjusted HR } \\
(95 \% \mathrm{Cl})\end{array}$ & $P$ value & $\begin{array}{c}\text { Adjusted HR } \\
(95 \% \mathrm{Cl})\end{array}$ & $P$ value \\
\hline \multicolumn{5}{|c|}{ Conservative group (DM patients, $n=621$; non-DM patients, $n=1,997$ ) } \\
\hline Aortic valve-related death and HF hospitalization & $1.33(1.15-1.54)$ & $<0.001$ & $1.33(1.13-1.56)$ & $<0.001$ \\
\hline Aortic valve-related death & $1.14(0.94-1.39)$ & 0.17 & $1.11(0.90-1.37)$ & 0.33 \\
\hline HF hospitalization & $1.30(1.10-1.54)$ & 0.002 & $1.36(1.14-1.63)$ & $<0.001$ \\
\hline All-cause death & $1.11(0.97-1.26)$ & 0.12 & $1.08(0.94-1.24)$ & 0.29 \\
\hline Cardiovascular death & $1.09(0.93-1.28)$ & 0.30 & $1.01(0.85-1.21)$ & 0.88 \\
\hline Sudden death & $1.45(1.04-2.01)$ & 0.03 & $1.17(0.82-1.67)$ & 0.40 \\
\hline \multicolumn{5}{|c|}{ DM subgroup (ITDM patients, $n=130$; non-ITDM patients, $n=491$ ) } \\
\hline Aortic valve-related death and HF hospitalization & $1.00(0.73-1.36)$ & 0.98 & $0.92(0.66-1.29)$ & 0.64 \\
\hline Aortic valve-related death & $1.07(0.71-1.61)$ & 0.76 & $1.04(0.67-1.61)$ & 0.87 \\
\hline HF hospitalization & $0.92(0.64-1.32)$ & 0.65 & $0.86(0.58-1.28)$ & 0.46 \\
\hline All-cause death & $1.21(0.93-1.59)$ & 0.16 & $1.09(0.81-1.45)$ & 0.57 \\
\hline Cardiovascular death & $1.29(0.93-1.78)$ & 0.13 & $1.17(0.82-1.66)$ & 0.39 \\
\hline Sudden death & $1.50(0.81-2.79)$ & 0.19 & $1.52(0.81-2.87)$ & 0.19 \\
\hline
\end{tabular}

$\mathrm{Cl}$, confidence interval; HR, hazard ratio. Other abbreviations as in Tables 1,2.

5-year incidences of AV-related death, HF hospitalization, cardiovascular death, all-cause death and sudden death between the ITDM and non-ITDM groups (Figure 5). In the multivariate cox regression analysis, the risk of ITDM relative to non-ITDM remained insignificant for any of the secondary outcomes (Table 3).

\section{Discussion}

The primary findings of this study were as follows: (1) in initially conservatively managed patients with severe AS, DM occurred in younger patients and those with higher BMI and BSA, and was associated with more comorbidities including hypertension, dyslipidemia, smoking, hemodialysis, and previous MI. The history of PCI or CABG was more common, whereas $\mathrm{V}_{\max }$, $\mathrm{mPG}$, and $\mathrm{EF}$ were lower in echocardiographic assessments in the DM group; (2) DM was associated with higher risk for the primary outcome measure, AV-related death and HF hospitalization; and (3) insulin use, however, was not related to a higher incidence of primary or secondary outcome measures.

Both DM and AS commonly occur in the elderly population; however, effects of DM on the long-term prognosis of patients with AS remains unclear. The present study analyzed 2,618 patients with severe AS who were managed with an initial conservative strategy, and $23.4 \%$ of them had DM. A composite of AV-related death and HF hospitalization was selected as the primary outcome, and the 5 -year incidence of that was significantly higher in DM patients compared with non-DM patients. The cumulative 5-year incidence of HF hospitalization was also significantly higher in DM patients than in non-DM patients. These results are consistent after adjusting for various confounding factors. Our results were supported by Lindman et a ${ }^{17}$ who investigated the effect of DM on left ventricle function and remodeling in AS patients. DM was associated with hypertrophic left ventricular remodeling, increased left ventricle mass, left ventricle end-systolic dimension, and reduced systolic function, indicating that DM induced adverse effects on cardiac function. Similar results were observed by Falcão-Pires et al, ${ }^{18}$ who found that AS patients with DM had significantly more impaired

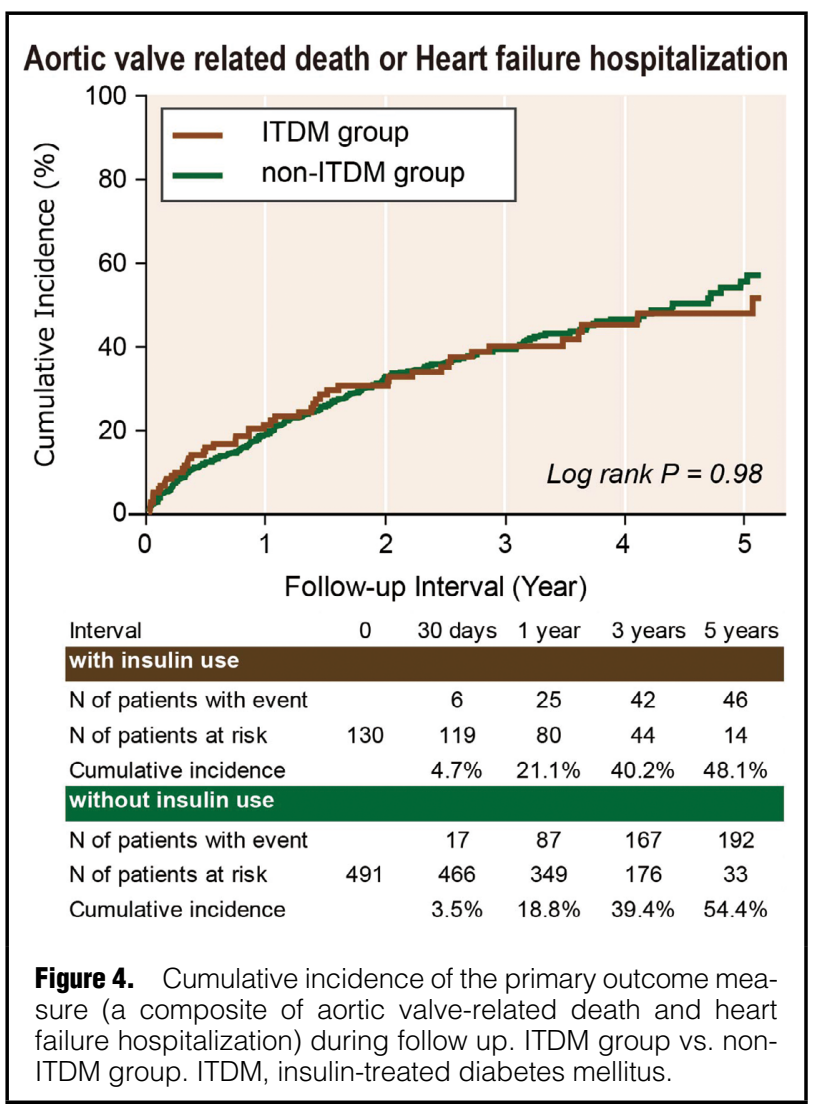

left ventricle diastolic function. Furthermore, DM accelerates atherosclerosis and is associated with increased risk of coronary artery disease. In the present study, patients with DM had more previous MI, thus, more had history of PCI and CABG. Therefore, DM directly impairs both myocardial diastolic and systolic functions and indirectly affects cardiac function by comorbidities like coronary artery disease. By these mechanisms, DM increases the risk of HF in patients with AS. 

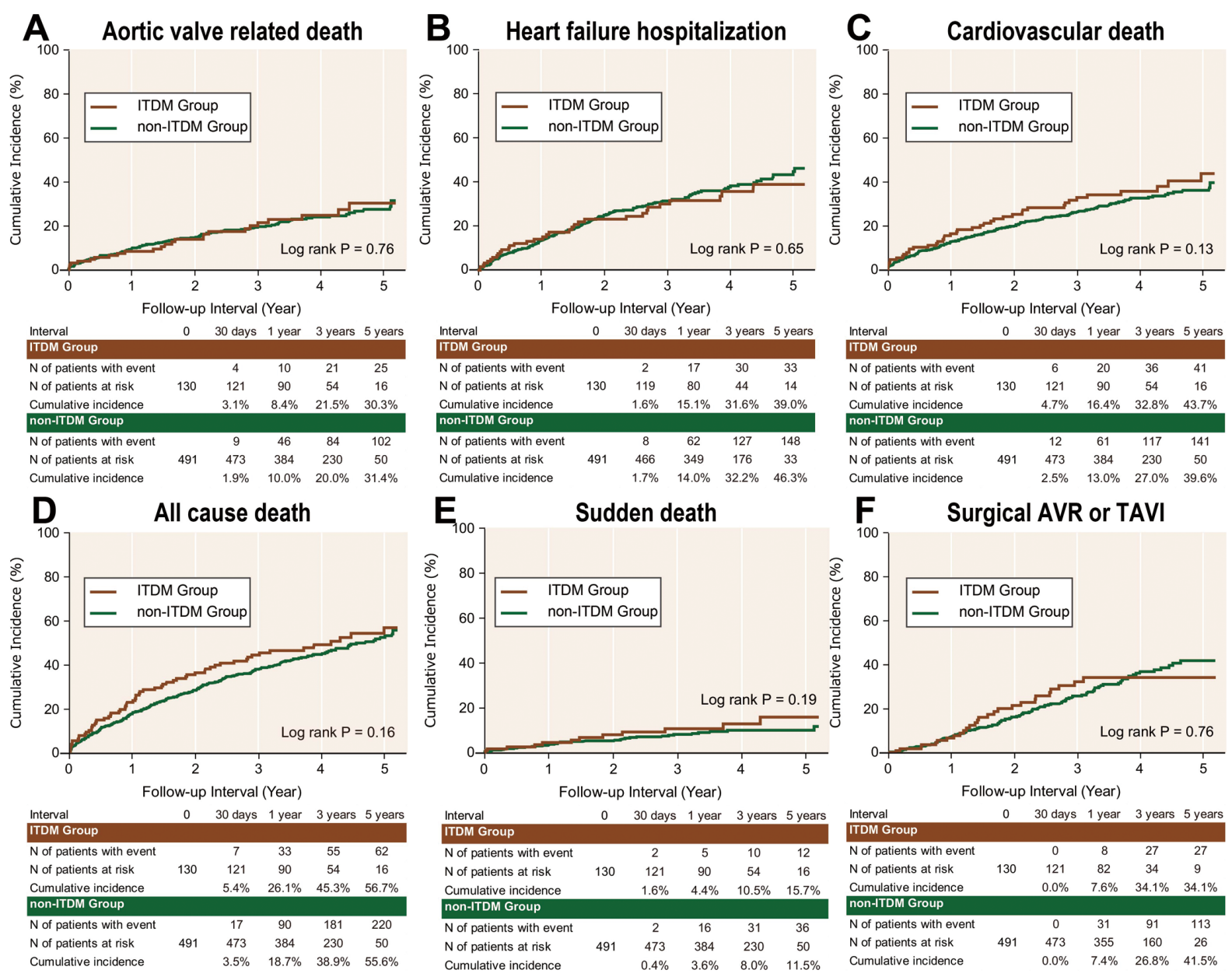

Figure 5. Cumulative incidence of secondary outcome measures including (A) aortic valve-related death, (B) heart failure hospitalization, (C) cardiovascular death, (D) all-cause death, (E) sudden death, and (F) surgical AVR or TAVI during follow up. ITDM group vs. non-ITDM group. ITDM, insulin-treated diabetes mellitus; AVR, aortic valve replacement; TAVI, transcatheter aortic valve implantation.

Another important question about the relationship between DM and AS is whether DM accelerates the AS progression. Some cross-sectional observational studies revealed that DM is associated with increased risk of $\mathrm{AV}$ calcification..$^{7,8}$ However, opinions on whether DM accelerates the progression of $\mathrm{AS}^{\mathbf{6 , 9 , 1 9}}$ or not $^{\mathbf{1 0}}$ varied. Nishimura et $\mathrm{al}^{3}$ investigated the progression of 140 asymptomatic patients with severe AS; the results showed that DM was an independent risk factor for first-year progression of $\mathrm{V}_{\max }$ and aortic valve area (AVA). In the CANHEART study, ${ }^{19}$ which involved 1.12 million Canadians, an independent relationship between DM and developing severe AS was found. Meanwhile, Testuz et a ${ }^{10}$ showed that DM caused no effect on AS progression in patients with at least mild AS. Although in this study, echocardiographic data were not available during the follow-up period, the 5-year incidence of SAVR or TAVI was higher in the DM group (40.1\% vs. $37.1 \%, \mathrm{P}=0.043)$, indicating a possible positive effect on AS progression by DM.

This study also examined the effect of insulin use on patients with DM, but as a result, insulin use failed to improve the primary or secondary outcome measures. The effect of insulin use on the cardiovascular outcome remains controversial. The United Kingdom Prospective Diabetes Study (UKPDS), which compared intensive care using insulin vs. standard care, showed reduction in the risk of microvascular events in the intensive control arm, but not for macrovascular events and all-cause mortality. Also, the very long follow up in the UKPDS showed a reduction of cardiovascular events. ${ }^{20}$ The DIGAMI 1 trial showed that intensive insulin infusion was associated with reduced allcause mortality, which was confirmed in a 20 -year post follow-up study; ${ }^{21}$ however, the DIGAMI 2 trial failed to show any benefit. ${ }^{22}$ Another landmark trial, the ACCORD study, was prematurely terminated due to high mortality in the intensive arm of the trial. ${ }^{23}$ The ORIGIN trial, which examined insulin glargine vs. standard care in 12,537 patients with DM, showed that early use of basal insulin neither increased nor reduced cardiovascular outcomes. ${ }^{24}$

Although there is a connection between DM and the incidence of AS, once the stenosis develops, DM may not affect $\mathrm{AS}$ in a similar way to atherosclerosis. ${ }^{4}$ This was 
supported by a randomized trial on statin; although lipidlowering therapies showed an improvement for the longterm outcome of atherosclerosis, and were thus used as the treatment for CAD; statins failed to delay the progression of severe AS, as reported by Cowell et al. ${ }^{25}$ Nevertheless, even when blood glucose (BG) was well-controlled by insulin therapy, the benefit may not be significant. In the ACCORD study, intensive therapy that targeted the HbAlc level below $6.0 \%$ showed no difference in a composite of non-fatal MI, non-fatal stroke, or death from cardiovascular causes, compared with a target level of $7.0-7.9 \%$. In addition, intensive therapy led to more hypoglycemia and higher all-cause mortality, thus the benefit of BG control was offset. ${ }^{23}$ Recently, several randomized controlled studies showed that the sodium-glucose co-transporter 2 (SGLT2) inhibitor reduced the risk of $\mathrm{HF}$ and cardiovascular mortality, and improved the outcome of patients with CAD. ${ }^{26-28}$ Although the mechanisms of these SGLT2 inhibitors are not fully understood, the proposed potential mechanisms include increased natriuresis, reduced blood pressure, renal protection and a modest effect to increase circulating ketones, which might improve myocardial energetics. In addition, the incidence of hypoglycemia was rare as reported by clinical study. ${ }^{29}$ However, no studies have ever confirmed the effects of the SGLT2 inhibitor on patients with AS. Future research evaluating the efficacy of the SGLT2 inhibitor on patients with AS is warranted.

\section{Study Limitations}

This study has several important limitations. First, this study was performed retrospectively without randomization; the decision about the initial treatment strategy was based on physicians' discretion. Second, detailed examination of echocardiographic data during follow up was not available, which made the assessment of AS progression difficult. Third, this study only included patients who did not receive initial TAVI or SAVR, which limits the generalizability of the study. Fourth, the CURRENT AS study was not focused on DM initially; therefore, data such as $\mathrm{HbA} 1 \mathrm{c}$ and duration of DM were not completely recorded, and the criteria for DM diagnosis were not fully in compliance with the guidelines. Finally, because the CURRENT AS registry included exclusively Japanese patients, this limits the application of study findings to other populations of Japan.

\section{Conclusions}

In initially conservatively managed patients with severe AS, DM was an independent risk factor for primary outcome measure, AV-related death and HF hospitalization; however, insulin use was not associated with poor outcomes.

\section{Sources of Funding}

This study was supported by the Research Institute for Production Development, Kyoto, Japan.

\section{Disclosures}

T. Kimura is one of the Associate Editors for Circulation Journal. The authors declare no other related conflicts of interest.

\section{IRB Information}

This study was approved by the Ethics Committee in Kyoto University, Kyoto, Japan. ID: E1916.

\section{Data Availability}

1. Will the individual deidentified participant data (including data dictionaries) be shared? Yes.

2. What data in particular will be shared? The deidentified participant data will be available on request.

3. Will any additional, related documents will be available? Yes, related documents will be available (e.g., statistical report).

4 When will the data become available and for how long? We will provide the requested data as soon as possible.

5. By what access criteria will the data be shared? Please contact the corresponding author for detailed information.

6. For what types of analyses, and by what mechanism will the data be available?

The analyses related to this study will be available. The mechanism is not decided yet. Please contact the corresponding author for further details.

\section{References}

1. López-de-Andrés A, Perez-Farinos N, de Miguel-Díez J, Hernández-Barrera V, Méndez-Bailón M, de Miguel-Yanes JM, et al. Impact of type 2 diabetes mellitus in the utilization and inhospital outcomes of surgical aortic valve replacement in Spain (2001-2015). Cardiovasc Diabetol 2018; 17: 135.

2. Mack MJ, Leon MB, Thourani VH, Makkar R, Kodali SK, Russo M, et al. Transcatheter aortic-valve replacement with a balloon-expandable valve in low-risk patients. $N$ Engl $J$ Med 2019; 380: 1695-1705.

3. Nishimura $S$, Izumi $C$, Nishiga $M$, Amano $M$, Imamura $S$, Onishi $\mathrm{N}$, et al. Predictors of rapid progression and clinical outcome of asymptomatic severe aortic stenosis. Circ J 2016; 80: 1863-1869.

4. Banovic M, Athithan L, McCann GP. Aortic stenosis and diabetes mellitus: An ominous combination. Diab Vasc Dis Res 2019; 16: 310-323.

5. Stamler J, Vaccaro O, Neaton JD, Wentworth D. Diabetes, other risk factors, and 12-yr cardiovascular mortality for men screened in the Multiple Risk Factor Intervention Trial. Diabetes Care 1993; 16: $434-444$.

6. Natorska J, Wypasek E, Grudzień G, Sobczyk D, Marek G, Filip G, et al. Does diabetes accelerate the progression of aortic stenosis through enhanced inflammatory response within aortic valves? Inflammation 2012; 35: 834-840.

7. Katz R, Wong ND, Kronmal R, Takasu J, Shavelle DM, Probstfield $\mathrm{JL}$, et al. Features of the metabolic syndrome and diabetes mellitus as predictors of aortic valve calcification in the multi-ethnic study of atherosclerosis. Circulation 2006; 113: 2113-2119.

8. Stewart BF, Siscovick D, Lind BK, Gardin JM, Gottdiener JS, Smith VE, et al. Clinical factors associated with calcific aortic valve disease. J Am Coll Cardiol 1997; 29: 630-634.

9. Kamalesh M, Ng C, El Masry H, Eckert G, Sawada S. Does diabetes accelerate progression of calcific aortic stenosis? Eur $J$ Echocardiogr 2009; 10: 723-725.

10. Testuz A, Nguyen V, Mathieu T, Kerneis C, Arangalage D, Kubota N, et al. Influence of metabolic syndrome and diabetes on progression of calcific aortic valve stenosis. Int J Cardiol 2017; 244: $248-253$.

11. Nishimura RA, Otto CM, Bonow RO, Carabello BA, Erwin JP, Guyton RA, et al. 2014 AHA/ACC Guideline for the Management of Patients With Valvular Heart Disease: Executive Summary: A report of the American College of Cardiology/American Heart Association Task Force on Practice Guidelines. Circulation 2014; 129: 2440-24492.

12. Lindman BR, Bonow RO, Otto CM. Current management of calcific aortic stenosis. Circ Res 2013; 113: 223-237.

13. Bernal E, Ariza-Solé A, Formiga F, Abu-Assi E, Carol A, Galián $\mathrm{L}$, et al. Conservative management in very elderly patients with severe aortic stenosis: Time to change? J Cardiol 2017; 69: 883887.

14. Bach DS, Siao D, Girard SE, Duvernoy C, McCallister BD, Gualano SK. Evaluation of patients with severe symptomatic aortic stenosis who do not undergo aortic valve replacement: The potential role of subjectively overestimated operative risk. Circ Cardiovasc Qual Outcomes 2009; 2: 533-539.

15. Taniguchi T, Morimoto T, Shiomi H, Ando K, Kanamori N, Murata K, et al. Initial surgical versus conservative strategies in patients with asymptomatic severe aortic stenosis. J Am Coll Cardiol 2015; 66: 2827-2838. 
16. Kappetein AP, Head SJ, Généreux P, Piazza N, van Mieghem $\mathrm{NM}$, Blackstone EH, et al. Updated standardized endpoint definitions for transcatheter aortic valve implantation: The Valve Academic Research Consortium-2 consensus document. J Thorac Cardiovasc Surg 2013; 145: 6-23.

17. Lindman BR, Arnold SV, Madrazo JA, Zajarias A, Johnson SN, Pérez JE, et al. The adverse impact of diabetes mellitus on left ventricular remodeling and function in patients with severe aortic stenosis. Circ Hear Fail 2011; 4: 286-292.

18. Falcão-Pires I, Hamdani N, Borbély A, Gavina C, Schalkwijk CG, Van Der Velden J, et al. Diabetes mellitus worsens diastolic left ventricular dysfunction in aortic stenosis through altered myocardial structure and cardiomyocyte stiffness. Circulation 2011; 124: $1151-1159$.

19. Yan AT, Koh M, Chan KK, Guo H, Alter DA, Austin PC, et al. Association between cardiovascular risk factors and aortic stenosis: The CANHEART Aortic Stenosis Study. J Am Coll Cardiol 2017; 69: 1523-1532.

20. Lund SS, Rossing P, Vaag AA. Follow-up of intensive glucose control in type 2 diabetes. $N$ Engl J Med 2009; 360: 416-418.

21. Ritsinger V, Malmberg K, Mårtensson A, Rydén L, Wedel H, Norhammar A. Intensified insulin-based glycaemic control after myocardial infarction: Mortality during 20 year follow-up of the randomised Diabetes Mellitus Insulin Glucose Infusion in Acute Myocardial Infarction (DIGAMI 1) trial. Lancet Diabetes Endocrinol 2014; 2: 627-633.

22. Malmberg K, Rydén L, Wedel H, Birkeland K, Bootsma A, Dickstein K, et al. FASTTRACK intense metabolic control by means of insulin in patients with diabetes mellitus and acute myocardial infarction (DIGAMI 2): Effects on mortality and morbidity. Eur Heart J 2005; 26: 650-661.

23. Action to Control Cardiovascular Risk in Diabetes Study Group; Gerstein HC, Miller ME, Byington RP, Goff DC Jr, Bigger JT, Buse JB, et al. Effects of intensive glucose lowering in type 2 diabetes. $N$ Engl J Med 2008; 358: 2545-2559.

24. Gerstein HC, Bosch J, Dagenais GR, Díaz R, Jung H, Maggioni $\mathrm{AP}$, et al. Basal insulin and cardiovascular and other outcomes in dysglycemia. $N$ Engl J Med 2012; 367: 319-328.

25. Cowell SJ, Newby DE, Prescott RJ, Bloomfield P, Reid J, Northridge DB, et al. A randomized trial of intensive lipid-lowering therapy in calcific aortic stenosis. $N$ Engl J Med 2005; 352: $2389-2397$.

26. Zinman B, Wanner C, Lachin JM, Fitchett D, Bluhmki E, Hantel $\mathrm{S}$, et al. Empagliflozin, cardiovascular outcomes, and mortality in type 2 diabetes. $N$ Engl J Med 2015; 373: 2117-2128.

27. Neal B, Perkovic V, Mahaffey KW, De Zeeuw D, Fulcher G, Erondu N, et al. Canagliflozin and cardiovascular and renal events in type 2 diabetes. N Engl J Med 2017; 377: 644-657.

28. McMurray JJV, Solomon SD, Inzucchi SE, Kober L, Kosiborod MN, Martinez FA, et al. Dapagliflozin in patients with heart failure and reduced ejection fraction. $N$ Engl J Med 2019; 381: 1995-2008.

29. Chao EC, Henry RR. SGLT2 inhibition-A novel strategy for diabetes treatment. Nat Rev Drug Discov 2010; 9: 551-559.

\section{Supplementary Files}

Please find supplementary file(s);

http://dx.doi.org/10.1253/circj.CJ-20-0681 